\title{
Understanding and Exploiting the Interaction of Electron Beams With Low- dimensional Materials - From Controlled Atomic-level Manipulation to Circumventing Radiation Damage
}

T. Susi ${ }^{1}$, A. Mittelberger ${ }^{1}$, C. Kramberger ${ }^{1}$, C. Mangler ${ }^{1}$, C. Hofer ${ }^{1}$, T.J. Pennycook ${ }^{1}$, J. Kotakoski $^{1}$ and J. C. Meyer ${ }^{1}$

${ }^{1 .}$ University of Vienna, Faculty of Physics, Vienna, Austria

Interactions of energetic electrons with materials that result in permanent changes in the structure, often referred to as radiation damage, can be both a hindrance for imaging as well as a useful tool for manipulating matter at the smallest scales. This presentation is aimed to give an overview of the current state as well as recent new results for understanding and controlling the beam-matter interaction especially in 2D materials using transmission electron microscopy (TEM) and scanning-TEM (STEM).

Relevant doses for beam-induced structural changes can vary by many orders of magnitude. Radiolysis of organic molecules can happen at doses as low as $10 \mathrm{e}^{-} / \AA^{2}[1]$, while defect-free graphene in a lowenergy $(<80 \mathrm{keV})$ electron beam can be stable up to extremely high doses: $10^{8} \mathrm{e}^{-} / \AA^{2}$ in a TEM at $80 \mathrm{kV}$ without creating a defect [2], and even up to $10^{10} \mathrm{e}^{-} / \AA^{2}$ at $60 \mathrm{kV}$ in ultra-high vacuum. However, defects in the lattice are usually still highly mobile under the same irradiation. Vacancies in graphene can be displaced up to 100 times by beam-induced bond rotations without adding or removing atoms [3]. Similarly, silicon impurities in graphene can be displaced by the electron beam, as long as they remain 3-coordinated [4]. Phosphorus atoms show a similar stability as silicon [5], while nitrogen impurities in graphene are remarkably stable [6].

Displacements that conserve the number of atoms open a new route (besides the ejection of atoms) for the atomic-level definition of patterns in the structure. It appears conceivable that impurities can be shifted and maybe assembled into patterns. For Si impurities in graphene, a mechanism for a directed displacement of the impurity was identified in Ref. [4]. Based on this mechanism, we show in Fig. 1 the controlled displacement of a Si impurity over 5 steps: In each step, the electron beam was parked on the carbon atom next to the $\mathrm{Si}$, until the two atoms exchanged places. This displacement is possible until a carbon atom is eventually lost, leaving the Si impurity in a 4-coordinated configuration which is no longer mobile under $60 \mathrm{keV}$ electron irradiation.

The above-mentioned configurations and their dynamics can be traced because the individual configuration is stable enough for a single atomic resolution exposure, which requires on the order of $10^{5} \mathrm{e}^{-} / \AA^{2}$. For imaging atomic configurations that are less beam-stable, we have developed an automated acquisition of large-area, low-dose data sets that achieves atomic resolution in nearly all exposures, and a maximum likelihood reconstruction algorithm that can recover effective high-dose views of frequently occurring deviations from the periodic lattice [7]. Fig. 2 shows an overview image (2a), a collection of $22 \times 22$ automatically acquired exposures from this area (2b), and an example of a single low-dose exposure (2c). The resolution can be verified from the Fourier transform (inset). From a large number of low-dose images of a sample with irradiation-induced defects, the lattice and frequently occurring defects could be reconstructed, even though the defects are not discernible in the individual exposures (Fig. 2d) [8]. 
References:

[1] RF Egerton, P Li, M Malac, Micron 35 (2004), p. 399.

[2] JC Meyer et al, Phys. Rev. Lett. 108 (2012), p. 196102.

[3] J Kotakoski, C Mangler, J Meyer, Nat. Comm. 5 (2014), p. 3991.

[4] T Susi et al, Phys Rev Lett 113 (2014) p. 115501.

[5] T Susi et al, 2D materials, in press (http://doi.org/10.1088/2053-1583/aa5e 78)

[6] JC Meyer et al, Nat. Mat. 10 (2011) p. 209; T. Susi et al, ACS Nano 6 (2012), p. 8837.

[7] C Kramberger, J Meyer, Ultramic. 170 (2016) p. 60; JC Meyer et al, Ultramic. 145 (2014) p. 13.

[8] The authors acknowledge funding from the European Research Council (ERC) project PICOMAT, the Austrian Science Fund (FWF) projects P25721-N20 and P 28322-N36, and the Wiener

Wissenschafts- Forschungs- und Technologiefonds (WWTF) via project MA14-009.
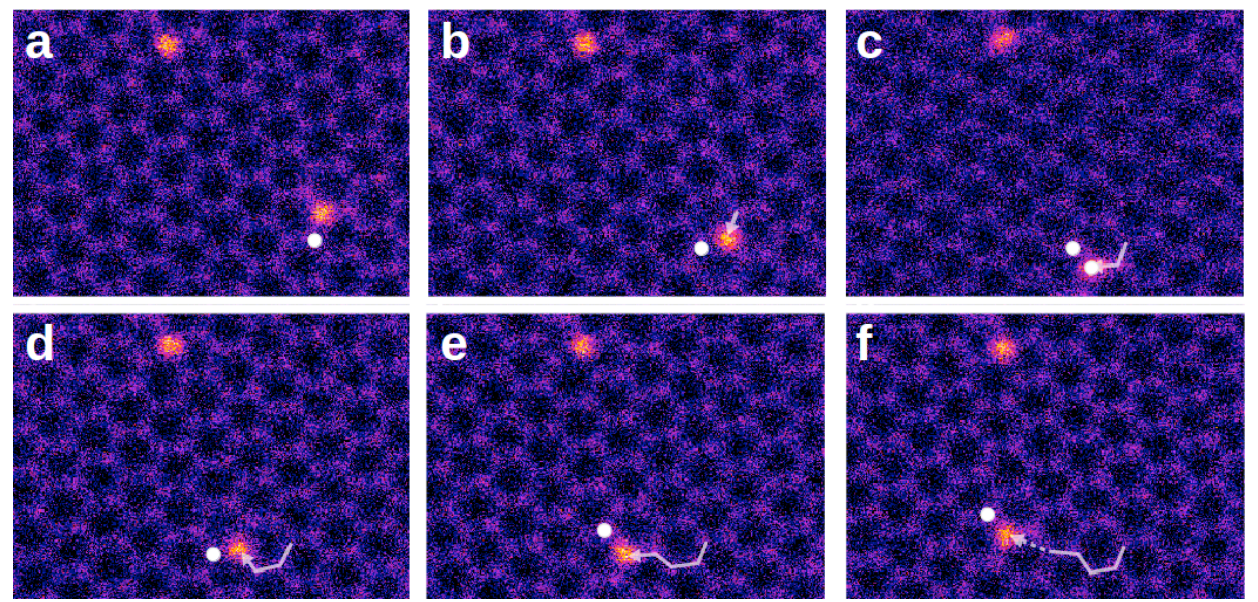

Figure 1. Controlled displacement of a Si impurity in graphene. In each step, the beam is parked on the position marked by the white dot in order to "pull" the Si atom to the new position ( $\mathrm{Si}$ and $\mathrm{C}$ exchange places). From e to $\mathrm{f}$, the Si moves further than intended. After f, a 4-fold configuration was obtained (corresponding to the loss of a carbon atom), which is no longer mobile. The Si atom at the top is also 4coordinated and serves as reference point.
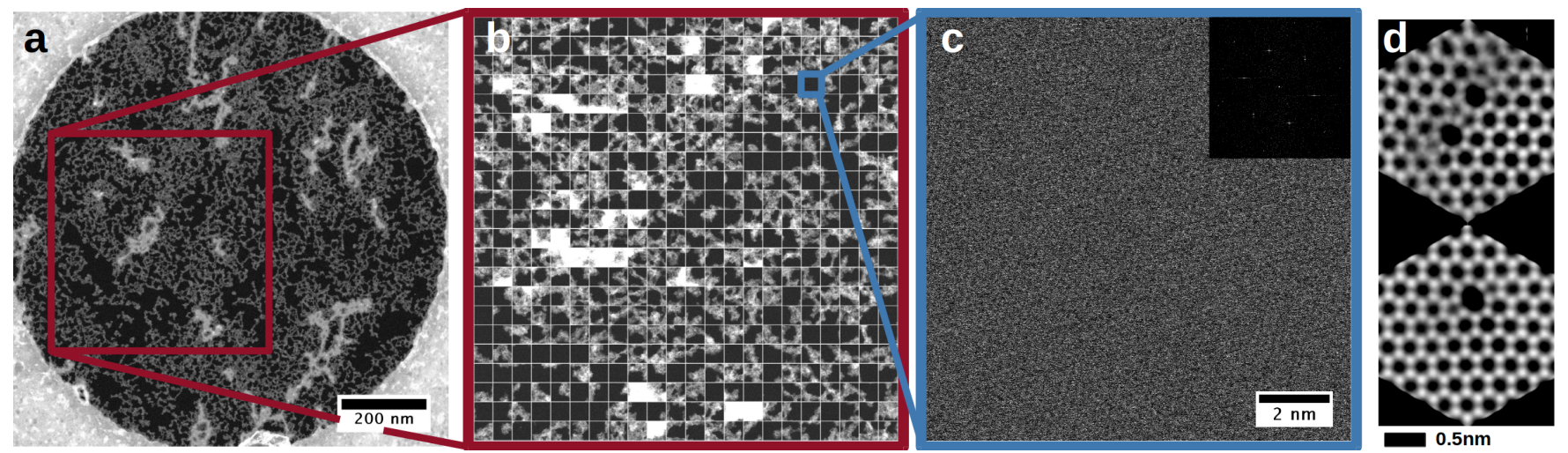

Figure 2. Automated large-area, low-dose acquisition. (a) Overview, (b) Collection of 22x22 images, (c) individual exposure, and Fourier transform (inset), (d) Results of a maximum likelihood reconstruction from a low-dose data set of a graphene sample with defects. 